\title{
Soft E-inversive Semigroup
}

\author{
Bingshen $\operatorname{Lan}^{1,} \mathrm{a}^{*}$ \\ ${ }^{1}$ The Engineering and Technical College of Chengdu University of Technology, Leshan, China \\ a512316180@qq.com
}

Keywords: Soft E-inversive semigroup; Soft normal semigroup; Soft group congruence

\begin{abstract}
In this paper, the concepts of Soft E-inversive semigroup, soft normal semigroup on E-inversive semigroup will be defined. Some results will be obtained. Finally, the concept of soft group congruence on Soft E-inversive semigroup is given, and the connections among soft group congruence and soft normal semigroup on E-inversive semigroup are revealed.
\end{abstract}

\section{Introduction}

1999, Molodstov gives soft set theory which is new mathematical tools deal with uncertainty problem in many fields [1]. After, soft set theory has been extended in various ways by many authors. Aktas $\mathrm{H}$, et al give the concept soft group. ${ }^{[2]}$, Ali discusses soft binary relationon and soft equivalence on set[3], Sezgin A, Atagün A O give the concept normalistic soft group[4] .

If $F: A \rightarrow P(U)$, then $(\mathrm{F}, \mathrm{A})$ is called soft set on $\mathrm{U} .[1]$

If $(\rho, A)$ is soft set on $U \times U$, then $(\rho, A)$ is called soft binary relation on $U \times U$.[3]

A Semigroup $\mathrm{S}$ with set $\mathrm{E}(\mathrm{S})$ of idempotents, if for any $s \in S_{\text {suah that }} \exists x \in S, s x \in E(S)$, then $\mathrm{S}$ will be called E-inversive semigroup.[5]

Let $\mathrm{S}$ be a semigroup, for any $s \in S$, if $x s x=x, x \in S$, then $\mathrm{x}$ is called a weak inverse of s. let $W(s)=\{x \in s: x s x=x\}$

denote all weak inverses of s. [6]

A Subsemigroup $\mathrm{T}$ of a E-inversive semigroup $\mathrm{S}$ is said to be unitary if for any $x, y \in S$,

(1) if $x+y \in S, x \in S$, then $y \in S$; and

(2) if $x+y \in S, y \in S$, then $x \in S$. [6]

A subsemigroup $\mathrm{T}$ of E-inversive semigroup $\mathrm{S}$ is said to be self-conjugate if for any $x \in S$, such that $x S x^{\prime} \in S, \quad x^{\prime} S x \in S, \quad x^{\prime} \in W(x)[6]$.

A subsemigroup $\mathrm{T}$ of E-inversive semigroup $\mathrm{S}$ is said to be noamal If $\mathrm{T}$ such that the following three conditions.

(1)T contains all the idempotents of $\mathrm{S}, \boldsymbol{E}(S) \subseteq \boldsymbol{T}$;

(2) $\mathrm{T}$ is unitary;

(3) T is self-conjugate. ${ }^{[6]}$

A binary relation $\rho$ on a set $\mathrm{X}$ (that is a subset of $X \times X$ ) is called a equivalence if

$(x, x) \in \rho$ for all $\mathrm{x}$ in $\mathrm{X}$, that is $\rho$ reflexive;

$\forall x, y \in X,(x, y) \in \rho \Rightarrow(y, x) \in \rho$, that is $\rho$ symmetric;

(3) $\forall x, y, z \in X,(x, y) \in \rho,(y, z) \in \rho \Rightarrow(x, z) \in \rho$, that is $\rho$ transitive. [7]

Let $\mathrm{S}$ be a semigroup. A binary relation $\rho$ on the set $\mathrm{S}$ is called left compatible (with the operation on $\mathrm{S}$ ) if 
$\forall s, t, a \in S,(s, t) \in \rho \Rightarrow(a s, a t) \in \rho$,

And right compatible if

$\forall s, t, a \in S,(s, t) \in \rho \Rightarrow(s a, t a) \in \rho$.

It is called compatible if

$\forall s, t, a, b \in S,(s, t) \in \rho,(a, b) \in \rho \Rightarrow(s a, t b) \in \rho$

A left (right) compatible equivalence is called a left (right) congruence. A compatible equivalence is called a congruence. ${ }^{[7]}$

\section{Soft E-Inversive Semigroup}

Definition 2.1 Let $(F, A)$ be a soft set on E-inversive semigroup $S$, for any $a$ of $A, F(a)$ is a E-inversive subsemigroup of $S$, then $(F, A)$ is called Soft E-inversive semigroup on $S$.

Example2.1 Let $\mathrm{S}=\{e, f, a, b\}$ be the E-inversive semigroup with multiplication table

\begin{tabular}{c|cccc} 
& e & f & a & b \\
\hline e & f & e & e & e \\
f & e & f & f & f \\
a & e & f & a & e \\
b & f & f & b & e
\end{tabular}

Let $A=\{\mathbf{1}, \mathbf{2}, \mathbf{3}, \mathbf{4}\}$, and let

$\mathrm{F}(1)=\{f\}, \mathrm{F}(2)=\{f, a\}, \mathrm{F}(3)=\{e, f, a\}, \mathrm{F}(4)=\{e, f, a, b\}$

It is easy to see that

$\mathrm{F}(1)=\{f\}, \mathrm{F}(2)=\{f, a\}, \mathrm{F}(3)=\{e, f, a\}, \mathrm{F}(4)=\{e, f, a, b\}$

are E-inversive subsemigroups of $S$. So, $(F, A)$ is Soft E-inversive semigroup on $S$.

Proposition2.1 If $(F, A),(H, A)$ be Soft E-inversive semigroup on E-inversive semigroup $S$, then $(F, A) \cap(H, A)$ is Soft E-inversive semigroup on $S$.

Proof. Let $(U, C)=(F, A) \bigcap(H, A), C=A$, for any $a$ of $A, U(a)=F(x)$, or $H(x)$, hence $(F, A) \bigcap(H, A)$ is Soft E-inversive semigroup as required.

Proposition 2.2 If $(F, A),(H, B)$ be Soft E-inversive semigroup on E-inversive semigroup $S$, $A \cap B=\Phi$, then $(F, A) \bigcup(H, B)$ is Soft E-inversive semigroup on $S$.

Proof Let $(U, C)=(F, A) \bigcup(H, B)$, for any $a$ of $C$, then $a \in A-B$ or $a \in B-A$, If $a \in A-B, \mathrm{U}(\mathrm{a})=\mathrm{F}(\mathrm{x})$, or $a \in B-A, U(a)=H(x)$, hence $(F, A) \bigcup(H, B)$ is Soft E-inversive semigroup as required.

Definition 2.2 Let $(F, A)$ be a soft set on E-inversive semigroup $S$, for any $a$ of $A, F(a)$ is a normal subsemigroup of $S$, then $(F, A)$ is called Soft normal semigroup on $S$.

Proposition2.3 If $(F, A)$ is Soft normal semigroup on E-inversive semigroup $\mathrm{S}$, then $(F, A)$ is $\operatorname{Soft}$ E-inversive semigroup on $S$.

Proof For any a of $A, F(a)$ is normal subsemigroup of $S$, then $E(S) \subseteq F(a), F(a)$ contains all the idempotents of $S$, and for any $s$ of $F(a)$, if $s x \in E(S), x \in S$, and $F(a)$ is unitary, it is clear that $x \in F(a)$, so $F(a)$ is E-inversive subsemigroup of $S$. hence (F,A) is soft E-inversive semigroup on $S$. 
Proposition2.4 If $(F, A),(H, B)$ are soft normal semigroup on E-inversive semigroup $S$, then $(F, A) \wedge(H, B)$ is Soft normal semigroup on $\mathrm{S}$.

Proof Let $(U, C)=(F, A) \wedge(H, B), \mathrm{C}=A \times B, U(\alpha, \beta)=F(\alpha) \cap H(\beta)$, for any $\alpha \in A, \beta \in B$, $E(S) \subseteq F(a), E(S) \subseteq H(\beta), E(S) \subseteq F(a) \bigcap H(\beta)$; and $F(\alpha), H(\beta)$ are self-conjugate, unitary, $U(\alpha, \beta)=F(\alpha) \cap H(\beta)$ is self-conjugate, unitary; Hence $U(\alpha, \beta)=F(\alpha) \cap H(\beta)$, is Soft normal semigroup as required.

\section{Soft Group Congruences on E-Inversive Semigroup}

If $\rho$ is a congruence on semigroup $S$, then we can define a binary operation on the quotient set $S / \rho$ as follows:

$(a \rho)(b \rho)=(a b) \rho \cdot[7]$

If $\rho$ is a congruence on E-inversive semigroup $S, S / \rho$ is a group, then $\rho$ is saided a group congruence on E-inversive semigroup $S$.

Definition3.1 $S$ is E-inversive semigroup, let $(\rho, A)$ be soft binary relation on $S \times S$, for any $\alpha \in A, \rho(\alpha)$ is a group congruence on a E-inversive semigroup $S$, then $(\rho, A)$ is called soft group congruence on E-inversive semigroup $S$.

Proposition3.1 let $N$ be normal subsemigroup of E-inversive semigroup $S$, then

$$
\rho_{N}=\{(a, b) \in S \times S \mid(\exists p, q \in N) p a=b q\}
$$

is a group congruence on $S$. Conversely, let $\rho$ be a a group congruence on E-inversive semigroup $S$, then

$$
N_{\rho}=\left\{a \in S \mid a \rho=1_{S / \rho}\right\}
$$

is a normal subsemigroup of E-inversive semigroup $\mathrm{S}[8]$.

Proposition3.2 let $(F$, A) is soft normal semigroup of E-inversive semigroup $S$, for any $a \in A$, let

$$
\rho(a)=\rho_{F(a)}=\{(a, b) \in S \times S \mid(\exists p, q \in F(a)) p a=b q\}
$$

Then ( $\rho$, A ) is soft group congruence on E-inversive semigroup $S$.

Conversely, let $(\rho, \mathrm{B})$ is soft group congruence on E-inversive semigroup $S$, then, for any $b \in B$, let

$$
F(b)=N_{\rho(b)}=\left\{s \in S \mid \mathrm{s} \rho=1_{S / \rho(b)}\right\}
$$

Then $(F, B)$ is soft normal semigroup of E-inversive semigroup $S$.

Proof If $(F, A)$ is soft normal semigroup of E-inversive semigroup $S$, so for any $a \in A, F(a)$ is normal subsemigroup of E-inversive semigroup $\mathrm{S}$,

Let, $\rho(a)=\rho_{F(a)}=\{(a, b) \in S \times S \mid(\exists p, q \in F(a)) p a=b q\}$

From Proposition3.1, $\rho(a)=\rho_{F(a)}=\{(a, b) \in S \times S \mid(\exists p, q \in F(a)) p a=b q\}$ is a group congruence on $S$. hence, ( $\rho, A)$ is soft group congruence on E-inversive semigroup $S$.

Conversely, if ( $\rho, \mathrm{B}$ ) is soft group congruence on E-inversive semigroup $S$, so any $b \in B, \rho$ (b) is group congruence on $\mathrm{S}$, let

$$
F(b)=N_{\rho(b)}=\left\{s \in S \mid \mathrm{s} \rho=1_{s / \rho(b)}\right\}
$$

From Proposition3.1,

$$
F(b)=N_{\rho(b)}=\left\{s \in S \mid \mathrm{s} \rho=1_{S / \rho(b)}\right\}
$$


is a normal subsemigroup of E-inversive semigroup $S$. hence, $(F, B)$ is soft normal semigroup of E-inversive semigroup $S$.

\section{References}

[1] Molodtsov D. Soft set theory-First results [J]. Comput Math Appl, 1999, 37(4):19-31.

[2] Aktas H, et al. Soft sets and soft groups [J]. Inform Sci, 2007,177 (13):2726-2735.

[3] Ali M I. A note on soft sets, rougf sets and fuzzy soft sets [J]. Applied Soft Computing, 2011, 11(4):3329-3332.

[4] Sezgin A, Atagün A O. Soft groups and normalistic soft groups [J]. Comput Math Appl, 2011, 62(2):685-698.

[5] Mistch H, Petrich M.Basic propositions of E-inversive semigroups. Commu Algebra, 2000 28:5169-5182.

[6] Fountain J B, Pin JE, and Weil P. Covers for monoids. J Algebra, 2004 271: 529-586.

[7] Howie J M. Fundamentals of Semigroup Theory [M]. Oxford: Clarendon Press, 1995.

[8] Lan Bingshen et al. Groupoid congruences of E-inversive semigroupoid. Journal of Sichuan Normal University (Natural Science), 2009, 5:564-568. 